\title{
Does the $\beta$-Blocker Nebivolol Increase Coronary Flow Reserve?
}

\author{
Mario Togni • Francesco Vigorito • Stephan Windecker • \\ Linda Abrecht $\cdot$ Peter Wenaweser $\cdot$ Stephane Cook . \\ Michael Billinger • Bernhard Meier • Otto M. Hess
}

Published online: 26 January 2007

(C) Springer Science + Business Media, LLC 2007 a significant increase in CFR due to reduction in resting flow (controls), or due to an increase in maximal coronary flow (CAD patients). CFI decreased with nebivolol parallel to the reduction in myocardial oxygen consumption.

Key words coronary artery disease $\cdot$ nebivolol . coronary flow reserve . coronary collateral flow

\section{Introduction}

The phenomenon of hypoxemia-induced increase in coronary flow was first described by Hilton and Eicholtz in 1925 [1]. Their findings were confirmed by Katz and Lindner in 1939 who reported a quantitative relation between reactive hyperemia and myocardial ischemia [2]. However, it was not until 1960 that the concept of coronary flow reserve was introduced by Coffman and Gregg [3] to assess the capacity of coronary circulation to conduct maximal hyperemic blood flow. Subsequently, coronary flow reserve has been used as a clinical parameter for assessing the functional significance of intermediate coronary stenoses. Three types of stimuli have been used to elicit maximal coronary blood flow in humans: (1) transient coronary occlusion during angioplasty (reactive hyperemia) [4]; (2) pharmacologic agents such as papaverine [5-7], adenosine [6-8], and dipyridamole [8]; (3) dynamic exercise [9].

Coronary flow reserve is defined as the ratio of coronary blood flow during maximal vasodilatation divided by resting flow, and measures the ability of the two components of myocardial perfusion, namely, epicardial stenosis resistance and microvascular resis-

F. Vigorito

Department of Cardiology, University of Napoli,

Napoli, Italy 
tance, to achieve maximal blood flow. In the absence of epicardial coronary artery lesions, the pressure-flow relationship is linear and steep during maximal vasodilatation and, therefore, peak flow is a function of coronary resistance [10]. Therapeutic interventions may affect coronary flow reserve by changing resting or maximal flow. A small increase in resting flow leads, however, to a large decrease in coronary flow reserve, and vice versa.

Beta-blockers reduce oxygen consumption of the myocardium and thus, diminish myocardial ischemia (=reduced demand). In addition, metoprolol has been shown to improve myocardial perfusion [11, 12] by increasing coronary flow reserve (=increased supply). This effect was mainly due to an increase in hyperemic flow rather than a decrease in resting flow. Nebivolol, a highly selective $\beta 1$-adrenergic receptor-blocker with nitric oxide-modulating properties [13], might be especially useful for improving coronary flow reserve due to its vasodilating properties on the small and large coronary arteries. Thus, the purpose of the present study was to evaluate the effect of intracoronary nebivolol on coronary flow velocity reserve and coronary flow reserve in controls and in patients with coronary artery disease using adenosine as stimulus for maximal flow increase. In addition, the effect of intracoronary nebivolol on collateral flow was assessed in patients with coronary artery disease during balloon inflation by calculating the collateral flow index.

\section{Methods}

Patient population Ten patients with angiographically normal coronary arteries (controls) and eight patients with one-vessel and one-lesion coronary artery disease (coronary artery disease group) were included in the present analysis (Table 1). Exclusion criteria were: untreated hypertension, know allergy or intolerance to beta-blocking agents, alcohol or drug abuse, renal insufficiency, clinically significant extracardiac disease, as well as diffuse or two and three vessel coronary artery disease. Diagnostic coronary angiography and left heart catheterization were performed with standard techniques. Aortic and left ventricular pressures were measured with a pigtail catheter. Biplane left ventricular angiography was performed at the end of diagnostic coronary angiography.

Stent implantation Of the eight coronary artery disease patients, three had an RCA lesion (two segment 2 and one segment 3), two an LAD lesion (both segment 13) and two an LCX lesion (both segment 20). Stent implantation was performed according to standard technique. Only bare-metal stents were implanted. Three patients received a BX-SONICTM stent (Cordis), three patients an AVE ${ }^{\mathrm{TM}}$ stent (Medtronic), and two patients a Vision ${ }^{\mathrm{TM}}$ (Guidant) stent. Lesion length was $8.1 \pm 2.1 \mathrm{~mm}$, preprocedure minimal lumen diameter was $0.57 \pm 0.45 \mathrm{~mm}$, mean stent length was $14 \pm 5 \mathrm{~mm}$, nominal stent diameter $3.0 \pm 0.2 \mathrm{~mm}$ and acute gain in-stent was $2.53 \pm 0.34 \mathrm{~mm}$.

Table 1 Patient characteristics

\begin{tabular}{|c|c|c|c|c|c|}
\hline & & Controls (\%) & $n$ & CAD (\%) & $p$ value \\
\hline Number of patients & 10 & - & 8 & & \\
\hline Age (year) & $57 \pm 6$ & - & $55 \pm 10$ & & 0.68 \\
\hline Men & 7 & $(70)$ & 6 & $(75)$ & 0.81 \\
\hline Smoking & 3 & $(30)$ & 6 & $(75)$ & 0.06 \\
\hline Diabetes mellitus & 2 & $(20)$ & 1 & $(12.5)$ & 0.67 \\
\hline Family history of CAD & 4 & $(40)$ & 4 & $(50)$ & 0.67 \\
\hline Hypertension & 6 & $(60)$ & 3 & $(37.5)$ & 0.34 \\
\hline Hypercholesterolemia & 7 & $(70)$ & 7 & $(87.5)$ & 0.38 \\
\hline Cholesterol (mmol/l) & $5.1 \pm 0.6$ & & $5.2 \pm 0.6$ & & 0.8 \\
\hline LVEDP (mmHg) & $11 \pm 3$ & & $10 \pm 2$ & & 0.58 \\
\hline LV-EF (\%) & $63 \pm 7$ & & $60 \pm 6$ & & 0.67 \\
\hline Aspirin & 7 & $(70)$ & 8 & $(100)$ & 0.09 \\
\hline Betablockers (metoprolol) & 5 & $(50)$ & 5 & $(62.5)$ & 0.6 \\
\hline Calcium antagonists & 1 & (10) & 1 & $(12.5)$ & 0.87 \\
\hline Lipid lowering agents & 7 & $(70)$ & 6 & (75) & 0.81 \\
\hline ACE inhibitors & 5 & $(50)$ & 8 & $(100)$ & 0.02 \\
\hline Nitrates & 1 & (10) & 3 & $(37.5)$ & 0.16 \\
\hline
\end{tabular}

$L V E D P$ Left ventricular end-diastolic pressure; $L V$-EF left ventricular ejection fraction; $C A D$ coronary artery disease 
Doppler-flow velocity measurements Doppler-flow velocity was measured with a 0.014 in. Doppler-guide wire with a $12-\mathrm{MHz}$ piezoelectric crystal at its tip (FloWire $^{\circledR}$, Volcano Therapeutics Inc., Rancho Cordova, CA, USA). The validation of this Doppler-guide wire has been described previously [14]. In coronary artery disease patients, the tip of the Doppler guidewire was placed distal to the stent. Coronary flow velocity reserve was calculated from hyperemic peak flow velocity averaged over two cardiac cycles (averaged peak velocity $=\mathrm{APV}, \mathrm{cm} / \mathrm{sec}$ ) divided by resting flow velocity. Pharmacologic modulation of coronary flow velocity reserve was induced by an intracoronary bolus of $18 \mu \mathrm{g}$ adenosine for the left and $12 \mu \mathrm{g}$ for the right coronary artery, respectively [7]. Coronary vascular resistance was calculated from mean aortic pressure divided by mean coronary flow velocity $(\mathrm{mmHg} \times \mathrm{sec} / \mathrm{cm})$. Coronary resistance ratio was calculated from coronary resistance during hyperemia divided by resistance at rest. Coronary flow was calculated from coronary flow velocity multiplied by cross-sectional area of the artery at the tip of the flow wire measured by quantitative coronary angiography using the ACA package of Philips DC/Integris system. Flow data were normalized by a factor of 0.5 assuming a parabolic flow profile and by a factor of 0.6 converting $\mathrm{cm} / \mathrm{sec}$ into $\mathrm{ml} / \mathrm{min}$ [14]. From these measurements coronary flow reserve was determined. In patients with coronary artery disease, after treatment of a flow-limiting stenosis with stent implantation, collateral flow velocity was calculated during subsequent balloon inflations. The collateral flow index (CFI) was determined as the ratio of the flow velocity time integral distal to the occluded artery during balloon inflation divided by baseline flow velocity obtained at the same location prior to balloon inflation.

\section{Study protocol}

1. Baseline: Vasoactive medication (including betablockers) was discontinued at least $48 \mathrm{~h}$ before catheterization. After coronary angiography and stent implantation for clinical purpose (only in coronary artery disease group), an interval of at least 15 min was allowed for dissipation of the effect of nonionic contrast medium. Two hundred micrograms of nitroglycerin was administered intracoronary for maximal vasodilation. Heart-rate, mean aortic pressure as well as coronary cross-sectional area at the tip of the flow wire were determined in all patients. Baseline coronary flow velocity and flow measurements were performed. Intracoronary adenosine was administered for assessment of coronary flow velocity and flow reserve, followed by a 1 min balloon inflation within stented segment (only in coronary artery disease group) for calculation of collateral flow index (Fig. 1).

\section{Study protocol}

\section{Stent *}

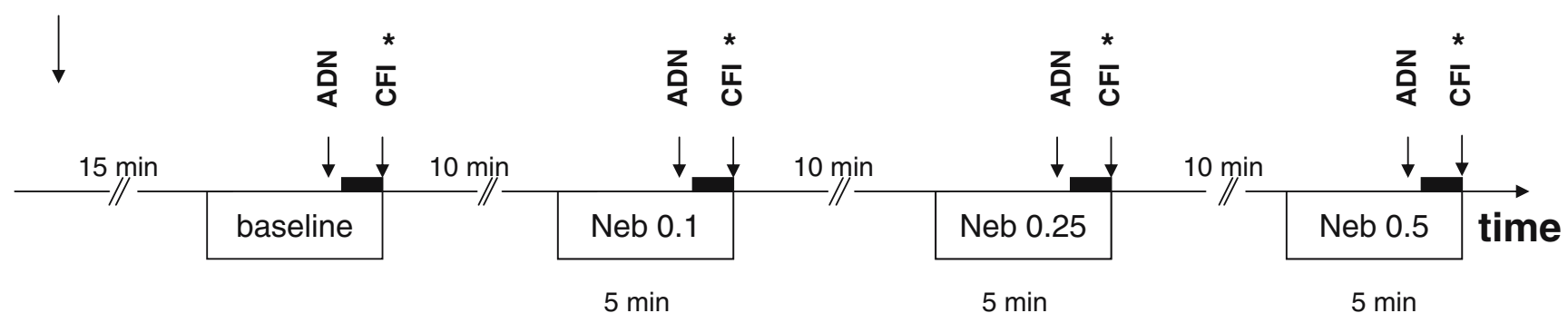

= vessel occlusion $1 \mathrm{~min}$ (CAD group only)

Fig. 1 Study protocol: After coronary angiography and stent implantation (only in CAD group), an interval of at least $15 \mathrm{~min}$ was allowed for dissipation of the effect of nonionic contrast medium. Two hundred micrograms of nitroglycerin was administered i.c. for maximal vasodilation. Baseline coronary flow velocity and flow measurements were performed. Intracoronary adenosine $(A D N)$ was administered for assessment of coronary flow velocity and flow reserve $(C F R)$, followed by a 1 min balloon inflation within stented segment (only in CAD group) for calculation of collateral flow index $(C F I)$. Three intracoronary nebivolol infusions were performed over a period of $5 \mathrm{~min}$ with increasing doses $(0.1,0.25$, and, $0.5 \mathrm{mg})$ separated by intervals of $10 \mathrm{~min}$ to allow coronary flow reach baseline level. At the end of each infusion period ADN was administered for CFR calculation followed by a 1 min balloon inflation (only in CAD group) for CFI calculation. Asterisk $=$ procedures and measurements performed only in CAD group 
2. Infusion of intracoronary nebivolol: Three intracoronary nebivolol infusions were administered over $5 \mathrm{~min}$ with increasing doses $(0.1,0.25$, and, $0.5 \mathrm{mg} / \mathrm{min})$ separated by intervals of $10 \mathrm{~min}$ to allow coronary flow reach baseline level. These doses adhere to comparable intra-arterial infusions into the brachial artery reported previously [15]. Doppler flow velocities were measured continuously. At the end of each infusion period, hyperemia was induced with adenosine for calculation of coronary flow and flow velocity reserve, followed by a $1 \mathrm{~min}$ balloon inflation (only in coronary artery disease group) for coronary flow index calculation (Fig. 2).

\section{Ethics}

The study protocol was approved by the local ethics committee, and written informed consent was obtained from all patients.

\section{Statistical analysis}

Comparison of angiographic, hemodymamic, and Doppler-flow velocity data was performed by an unpaired Student $t$-test (continuous variables ) and by a $\chi^{2}$-test (categorical variables). A two-way analysis of variance for repeated measurements was performed for comparison of hemodynamic and Doppler-flow data during nebivolol administration. Multivariable logistic regression analysis was performed to determine the effect of cardiovascular risk factors (hypertension, smoking) and baseline medication (ACE inhibitors and nitrates) as covariates on coronary flow velocity and coronary flow (including coronary flow velocity reserve and coronary flow reserve) response to nebivolol application. Correlation between coronary flow index and heart rate as well as rate-pressure product was performed using the square method. The correlation coefficient $(r)$ and the regression equation were calculated from these data. Statistical significance was defined at a $p$ value of $<0.05$.

\section{Results}

Baseline patient characteristics Patients in the two groups were well matched with regard to age, gender, presence of risk factors (diabetes mellitus, family history, and hypercholesterolemia) as well as medication (beta-blockers, calcium-antagonists). There was an imbalance with regard to history of smoking (higher incidence of smokers in the coronary artery disease group, $p=0.06$ ) and medication with ACE inhibitors (all coronary artery disease patients on ACE-inhibitor medication as opposed to only $50 \%$ in the control group, $p=0.02$ ) (Table 1).

Hemodynamics There was a significant decrease in heart rate after nebivolol administration in both groups. Mean aortic pressure remained unchanged after nebivolol in controls as well as in coronary

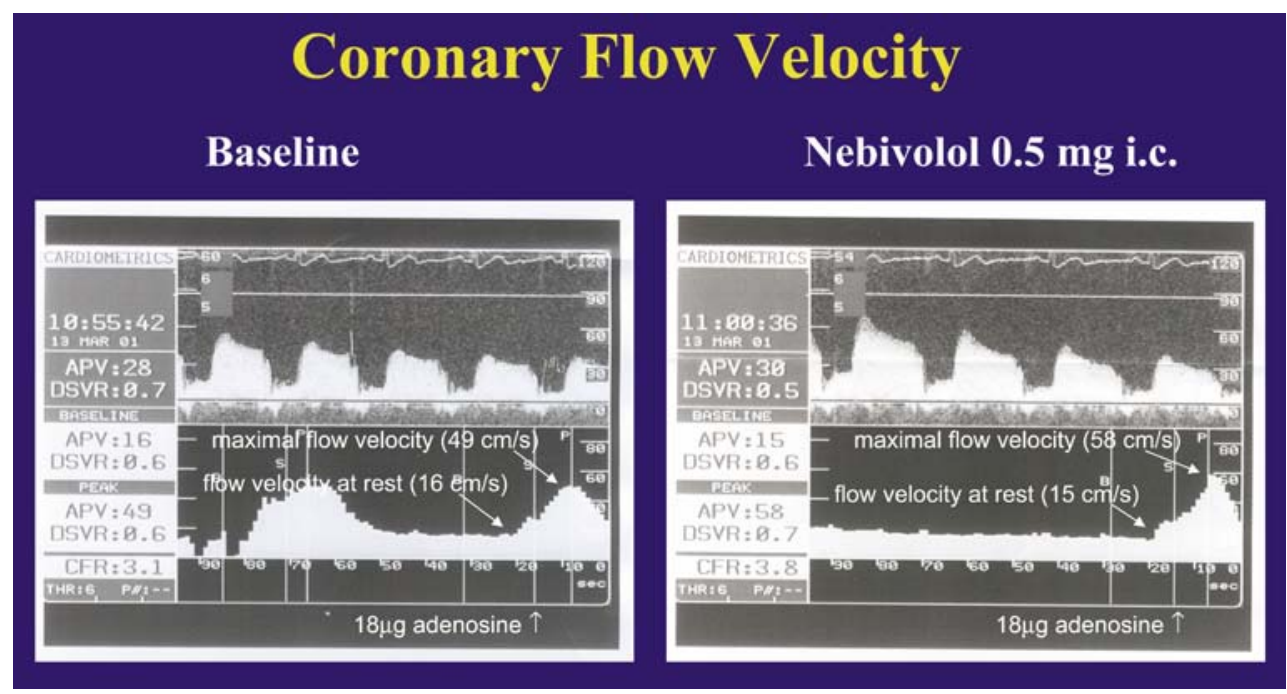

Fig. 2 Representative recordings of coronary flow velocity in a coronary artery disease patient before (left) and after $0.5 \mathrm{mg}$ intracoronary nebivolol (right). Coronary flow velocity at rest remained unchanged (from 16 to $15 \mathrm{~cm} / \mathrm{sec}$ after $0.5 \mathrm{mg}$ nebivolol), whereas maximal flow velocity increased from 49 to $58 \mathrm{~cm} / \mathrm{sec}$, respectively. As a consequence, coronary flow velocity reserve increased from 3.1 to 3.8 
Table 2 Hemodynamics

\begin{tabular}{|c|c|c|c|c|}
\hline & Baseline & Nebivolol $0.1 \mathrm{mg}$ & Nebivolol $0.25 \mathrm{mg}$ & Nebivolol $0.5 \mathrm{mg}$ \\
\hline \multicolumn{5}{|l|}{ Controls: } \\
\hline HR (beats/min) & $73 \pm 16$ & $68 \pm 15$ & $66 \pm 14 * *$ & $64 \pm 11 * *$ \\
\hline Systolic blood pressure $(\mathrm{mmHg})$ & $142 \pm 15$ & $143 \pm 14$ & $140 \pm 11$ & $139 \pm 11$ \\
\hline Diastolic blood pressure $(\mathrm{mmHg})$ & $82 \pm 8$ & $83 \pm 9$ & $80 \pm 8$ & $81 \pm 7$ \\
\hline $\mathrm{RPP}\left(\mathrm{mmHg} \times 10^{3} / \mathrm{min}\right)$ & 10.4 & 9.7 & 9.2 & $8.9 *$ \\
\hline \multicolumn{5}{|l|}{ CAD group: } \\
\hline HR (beats/min) & $71 \pm 9$ & $70 \pm 11$ & $65 \pm 7 *$ & $63 \pm 9 *$ \\
\hline Systolic blood pressure $(\mathrm{mmHg})$ & $140 \pm 21$ & $140 \pm 26$ & $135 \pm 17$ & $135 \pm 16$ \\
\hline Diastolic blood pressure $(\mathrm{mmHg})$ & $84 \pm 10$ & $82 \pm 11$ & $75 \pm 13$ & $76 \pm 14$ \\
\hline $\mathrm{RPP}\left(\mathrm{mmHg} \times 10^{3} / \mathrm{min}\right)$ & 9.9 & 9.8 & $8.8^{*}$ & $8.5^{*}$ \\
\hline
\end{tabular}

$H R$ Heart rate; $R P P$ rate-pressure product $* p<0.05, * * p<0.01$

artery disease patients. As a consequence, the ratepressure product (RPP) decreased after intracoronary nebivolol from $10.4 \times 10^{3}$ to $8.9 \times 10^{3} \mathrm{mmHg} / \mathrm{min}$ $(p<0.05)$ in controls and from $9.9 \times 10^{3}$ to $8.5 \times 10^{3}$ $\mathrm{mmHg} / \mathrm{min}(p<0.05)$ in coronary artery disease patients (Table 2).
Coronary flow velocity and flow reserve Coronary flow velocity reserve at rest was $3 \pm 0.6$ in controls and $2.1 \pm 0.4$ in coronary artery disease patients $(p<0.05)$. Coronary flow velocity reserve increased after intracoronary doses of $0.1,0.25$ and $0.5 \mathrm{mg}$ nebivolol to $3.1 \pm 0.6$ (ns), $3.5 \pm 0.8(p<0.05), 3.6 \pm 0.8(p<0.05)$ in controls and

\section{Coronary Flow Reserve}

\section{Controls}

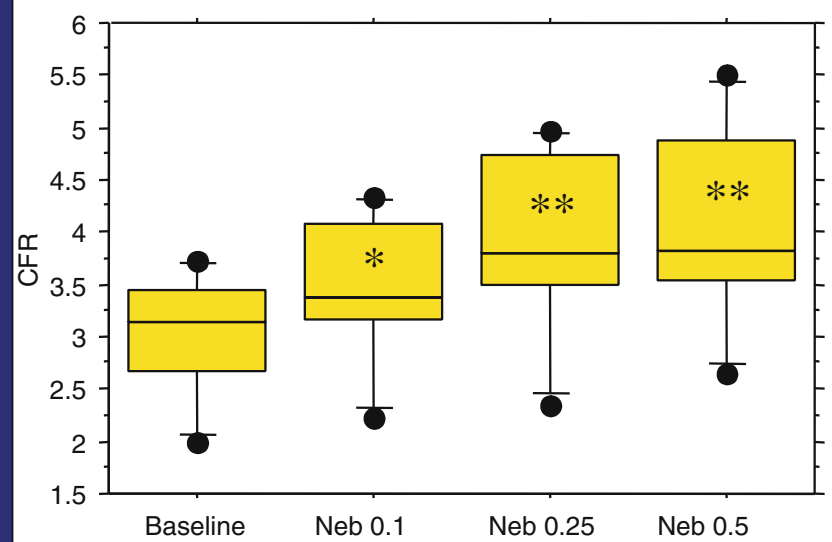

CAD

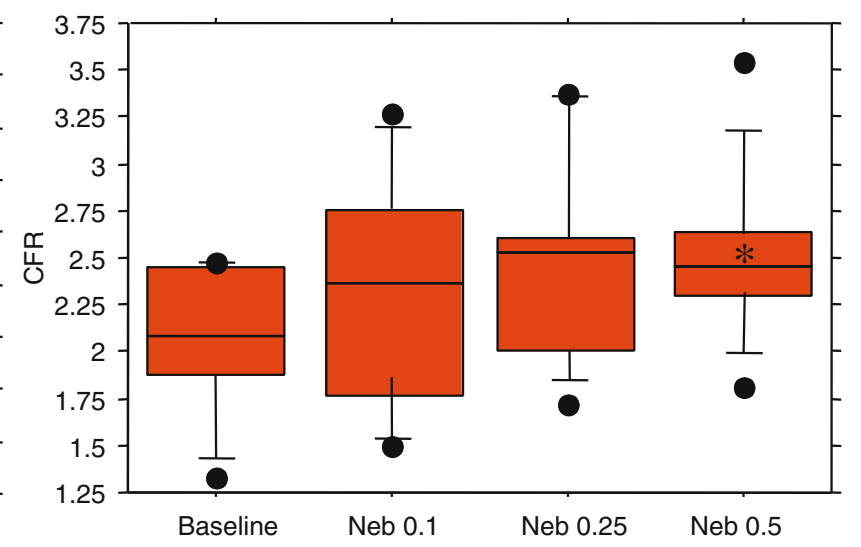

\section{$* p<0.05$ vs. baseline ; ** $p<0.01$ vs. baseline}

Fig. 3 Box plot of coronary flow reserve $(C F R)$ at rest and after intracoronary nebivolol in controls (left panel) and in CAD patients (right panel). CFR increased after intracoronary doses of $0.1,0.25$, and $0.5 \mathrm{mg}$ nebivolol from $3.0 \pm 0.6$ to $3.4 \pm 0.7(p<0.05)$,
$3.9 \pm 0.9(p<0.01)$ and $4.0 \pm 0.1 \quad(p<0.01)$ in controls and from $2.1 \pm 0.4$ to $2.3 \pm 0.7$ (ns), $2.6 \pm 0.9$ (ns), and $2.6 \pm 0.5(p<0.05)$ in CAD patients, respectively (abbreviations as in Fig. 1) 
Table 3 Coronary flow velocity

\begin{tabular}{|c|c|c|c|c|}
\hline & Baseline & Nebivolol $0.1 \mathrm{mg}$ & Nebivolol $0.25 \mathrm{mg}$ & Nebivolol $0.5 \mathrm{mg}$ \\
\hline \multicolumn{5}{|l|}{ Controls: } \\
\hline CFV rest $(\mathrm{cm} / \mathrm{sec})$ & $18 \pm 2$ & $16 \pm 2$ & $15 \pm 2 *$ & $14 \pm 2 * *$ \\
\hline CFV hyperemic $(\mathrm{cm} / \mathrm{sec})$ & $54 \pm 8$ & $50 \pm 7$ & $50 \pm 7$ & $49 \pm 7$ \\
\hline CFVR & $3 \pm 0.6$ & $3.1 \pm 0.6$ & $3.5 \pm 0.8^{*}$ & $3.6 \pm 0.8^{*}$ \\
\hline $\mathrm{CSA}\left(\mathrm{mm}^{2}\right)$ & $5.1 \pm 1.9$ & $5.0 \pm 1.8$ & $5.0 \pm 1.8$ & $5.0 \pm 1.7$ \\
\hline \multicolumn{5}{|c|}{ Coronary artery disease group: } \\
\hline $\mathrm{CFV}$ rest $(\mathrm{cm} / \mathrm{sec})$ & $24 \pm 7$ & $25 \pm 7$ & $26 \pm 7$ & $24 \pm 8$ \\
\hline CFV hyperemic (cm/sec) & $49 \pm 17$ & $54 \pm 13$ & $61 \pm 20$ & $56 \pm 13$ \\
\hline CFVR & $2.1 \pm 0.4$ & $2.2 \pm 0.7$ & $2.5 \pm 1$ & $2.4 \pm 0.6$ \\
\hline $\mathrm{CSA}\left(\mathrm{mm}^{2}\right)$ & $5.0 \pm 1.4$ & $5.3 \pm 1.4$ & $5.1 \pm 1.4$ & $5.2 \pm 1.3$ \\
\hline
\end{tabular}

$C F V$ Coronary flow velocity; $C F V R$ coronary flow velocity reserve; $C S A$ cross sectional area

$\sec ^{*} p<0.05 ; * * p<0.01$

to $2.2 \pm 0.7,2.5 \pm 1.0,2.4 \pm 0.6$ (all ns) in coronary artery disease patients, respectively. The similar pattern was observed for coronary flow reserve in both groups. Precisely, coronary flow reserve at rest was $3.0 \pm 0.6$ in controls and $2.1 \pm 0.4$ in coronary artery disease patients $(p<0.05)$. Coronary flow reserve increased after intracoronary doses of $0.1,0.25$, and $0.5 \mathrm{mg}$ nebivolol to $3.4 \pm 0.7(p<0.05), 3.9 \pm 0.9(p<0.01)$, and $4.0 \pm 0.1(p<0.01)$ in controls, and to $2.3 \pm 0.7$ (ns), $2.6 \pm 0.9$ (ns), and $2.6 \pm 0.5$ $(p<0.05)$ in coronary artery disease patients, respectively (Fig. 3) (Tables 3 and 4).

Coronary vascular resistance Coronary vascular resistance (CR) at rest increased after nebivolol in controls, whereas it did not change in coronary artery disease patients. Hyperemic coronary vascular resistance remained unchanged after nebivolol in controls, whereas it decreased significantly after $0.25 \mathrm{mg}(p<0.05)$ and $0.5 \mathrm{mg}(p<0.05)$ intracoronary nebivolol in coronary artery disease patients. Coronary resistance ratio at rest was $0.34 \pm 0.8$ in controls and $0.5 \pm 0.1$ in coronary artery disease patients, and decreased with $0.5 \mathrm{mg}$ nebivolol to $0.29 \pm 0.6$ in controls $(p<0.01)$ and to $0.45 \pm 0.1(\mathrm{~ns})$ in coronary artery disease patients, respectively (Table 5).
Collateral flow index Coronary flow index decreased from $0.37 \pm 0.23$ to $0.33 \pm 0.25,0.28 \pm 0.3 \quad(p<0.05)$, and $0.27 \pm 0.30(p<0.05)$, respectively after $0.1,0.25$, and 0.5 $\mathrm{mg}$ intracoronary nebivolol. There was a significant correlation between heart-rate and coronary flow index $(r=0.76, p<0.0001)$, as well as between ratepressure product and coronary flow index $(r=0.59$, $p<0.001$ ), respectively (Figs. 4 and 5).

\section{Discussion}

The present study shows that: (1) Intracoronary nebivolol is associated with a significant increase in both coronary flow velocity reserve and coronary flow reserve in patients with angiographically normal coronary arteries due to a reduction in resting but no change in maximal flow. (2) In coronary artery disease patients, coronary flow velocity reserve and coronary flow reserve increase after nebivolol due to an increase in maximal but no change in resting coronary flow. (3) Collateral flow decreases after nebivolol due to the reduction in myocardial oxygen requirements.

Table 4 Coronary flow

\begin{tabular}{lllll}
\hline & Baseline & Nebivolol $0.1 \mathrm{mg}$ & Nebivolol $0.25 \mathrm{mg}$ & Nebivolol $0.5 \mathrm{mg}$ \\
\hline Controls: & & & & $4.0 \pm 1^{* *}$ \\
CFR & $3 \pm 0.6$ & $3.4 \pm 0.7 *$ & $3.9 \pm 0.9 * *$ & $111 \pm 24$ \\
CF rest (ml/sec) & $113 \pm 31$ & $126 \pm 45$ & $121 \pm 30$ & $277 \pm 54^{*}$ \\
CF hyperemic(ml/sec) & $239 \pm 83$ & $276 \pm 78$ & $297 \pm 79$ & $2.6 \pm 0.5^{*}$ \\
Coronary artery disease group: & & & $2.6 \pm 0.9$ & $63 \pm 27 * *$ \\
CFR & $2.1 \pm 0.4$ & $2.3 \pm 0.7$ & $68 \pm 30^{*}$ & $236 \pm 80$ \\
CF rest (ml/sec) & $83 \pm 35$ & $74 \pm 29$ & $244 \pm 84$ & \\
CF hyperemic $(\mathrm{ml} / \mathrm{sec})$ & $236 \pm 80$ & $240 \pm 81$ & & \\
\hline
\end{tabular}

$C F$ Coronary flow; $C F R$ coronary flow reserve ${ }^{*} p<0.05 ;{ }^{* *} p<0.01$ 
Table 5 Coronary resistance

\begin{tabular}{lllll}
\hline & Baseline & Nebivolol $0.1 \mathrm{mg}$ & Nebivolol $0.25 \mathrm{mg}$ & Nebivolol $0.5 \mathrm{mg}$ \\
\hline Controls: & & & & $7.5 \pm 0.5^{*}$ \\
$\mathrm{CR}$ rest $(\mathrm{mmHg} \times \mathrm{sec} / \mathrm{cm})$ & $5.6 \pm 0.8$ & $6.3 \pm 0.6$ & $6.9 \pm 0.6$ & $2.1 \pm 0.6$ \\
CR hyperemic $(\mathrm{mmHg} \times \mathrm{sec} / \mathrm{cm})$ & $2.0 \pm 0.6$ & $2.1 \pm 0.6$ & $2.0 \pm 0.5$ & $0.29 \pm 0.6^{* *}$ \\
CRR & $0.34 \pm 0.8$ & $0.34 \pm 0.7$ & $0.30 \pm 0.7 * *$ & $4 \pm 1.4$ \\
Coronary artery disease group: & & & & $1.7 \pm 0.4^{*}$ \\
CR rest $(\mathrm{mmHg} \times \mathrm{sec} / \mathrm{cm})$ & $4.6 \pm 1.7$ & $4.8 \pm 2.1$ & $1.8 \pm 0.6^{*}$ & $0.45 \pm 0.1$ \\
CR hyperemic $(\mathrm{mmHg} \times \mathrm{sec} / \mathrm{cm})$ & $2.2 \pm 0.6$ & $2.1 \pm 0.7$ & $0.47 \pm 0.1$ & \\
CRR & $0.5 \pm 0.1$ & $0.47 \pm 0.2$ & \\
\hline
\end{tabular}

$C R$ Coronary resistance; $C R R$ coronary resistance ratio $* p<0.05 ; * * p<0.01$

Changes in coronary hemodynamics associated with the administration of $\beta$-blockers have been extensively studied in the past [16]. Previous clinical studies using coronary flow measurements suggest that non-selective $\beta$-adrenergic antagonists are associated with an increase in coronary vascular resistance during hyperemia and, therefore, reduce coronary flow reserve $[17,18]$. This phenomenon has been attributed to the unopposed alpha-adrenergic vasomotor tone. Selective $\beta$-adrenergic antagonists on the other hand are associated with an increase of coronary flow reserve [11]. The increase in coronary flow reserve with metoprolol administration is achieved by an increase in maximal coronary flow which is further enhanced by a decrease in resting flow $[11,12]$. With metoprolol, coronary vascular resistance decreases during adenosine-induced hyperemia.

Nebivolol combines potent $\beta 1$-adrenoreceptorblocking activity with nitric oxide-mediated vasodilating properties. In vitro studies have shown that

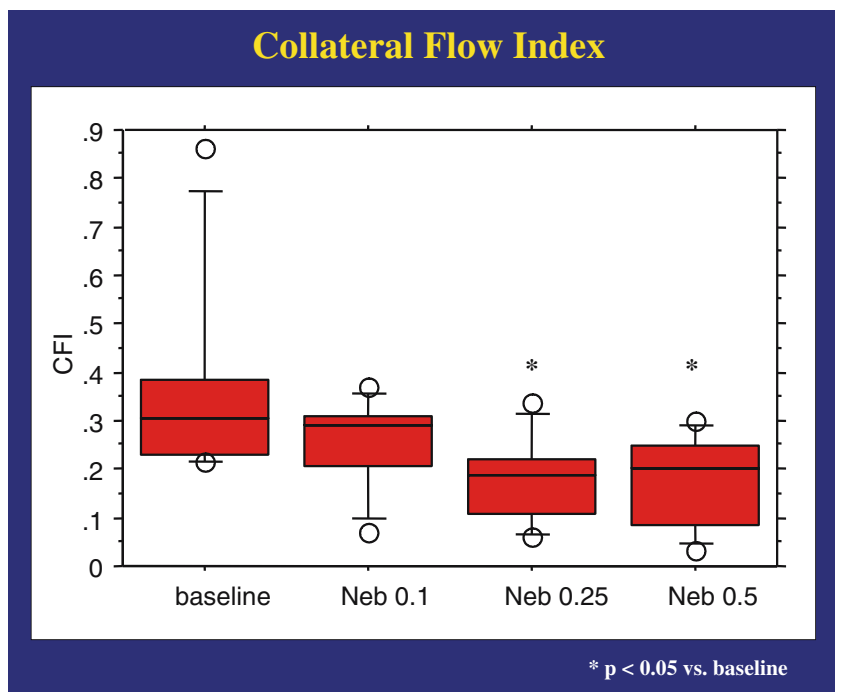

Fig. 4 Box plot of collateral flow index $(C F I)$ at rest and after intracoronary nebivolol in CAD patients. CFI decreased from $0.37 \pm 0.23$ to $0.33 \pm 0.25,0.28 \pm 0.3(p<0.05)$, and $0.27 \pm 0.3(p<0.05)$, respectively, after $0.1,0.25$, and $0.5 \mathrm{mg}$ of intracoronary nebivolol nebivolol dilates coronary resistance vessels in humans through an agonist effect on endothelial $\beta 3$-adrenoreceptors to release nitric oxide and promote neoangiogenesis [19]. The nitric oxide-releasing and vasodilating properties of nebivolol in coronary microvessels may exert its beneficial effect in patients with ischemic and dilated cardiomyopathies [20], particularly those with diastolic dysfunction [21], given the direct lusitropic properties of nitric oxide on the myocardium. Diseases such as dyslipidemia, diabetes, and atherosclerosis are all associated with a decrease in eNOS expression, nitric
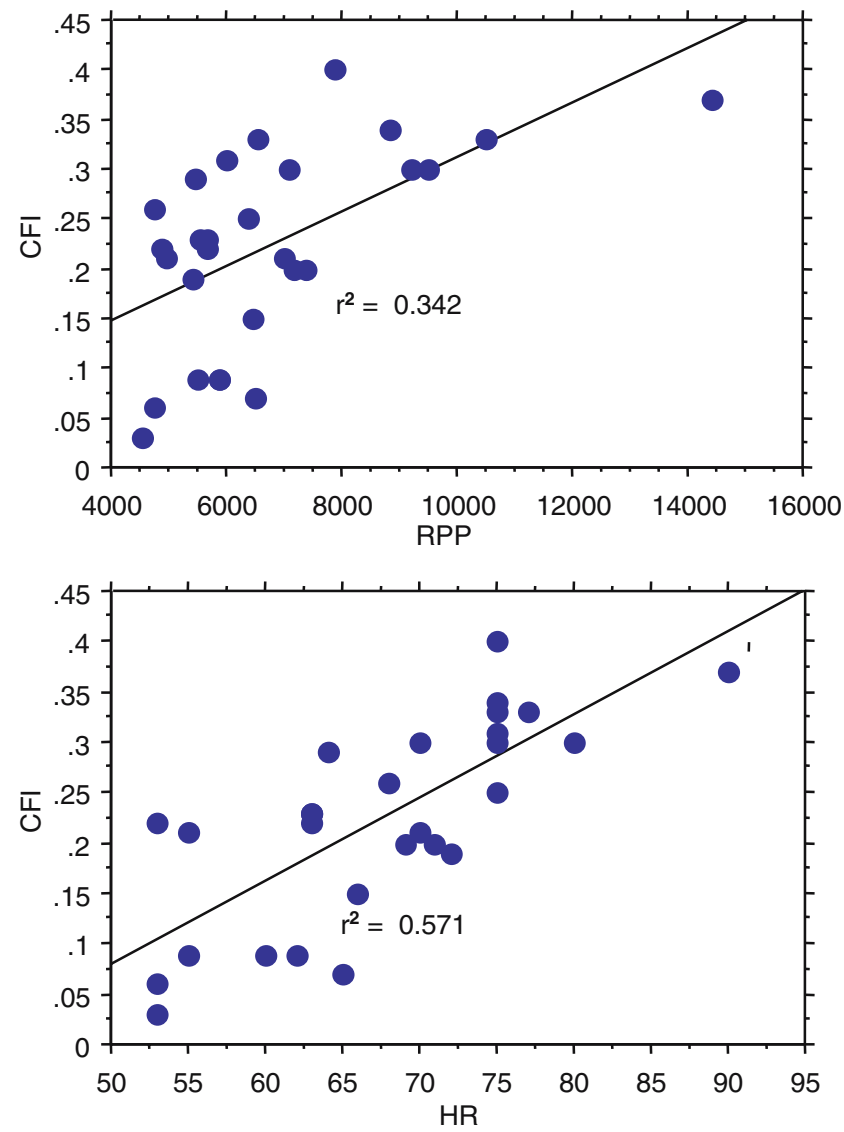

Fig. 5 Regression plot of collateral flow index versus heart rate (top panel) and rate-pressure product (lower panel), respectively 
oxide bioavailability, or both, which could limit the clinical impact of a drug that depends on nitric oxide release. The marked vasodilating effect of nebivolol in human coronary microvessels is well established under in vitro conditions [13]. The present study compliments a recent in vivo investigation by Galderisi et al. who demonstrated improvement of coronary flow reserve in hypertensive patients without coronary artery disease [22]. Nebivolol increased coronary flow reserve through an increase in hyperemic flow without changing resting flow comparable to our findings in the coronary artery disease patients. However, the two studies differ substantially. In the work by Galderisi: (1) exclusively hypertensive subjects without coronary artery disease were investigated, (2) coronary flow reserve was assessed by non-invasive means (Doppler echocardiography), and (3) nebivolol was administered by the oral route over 4 weeks. Importantly, Galderisi was able to demonstrate that adequate nebivolol concentrations are reached in the coronary blood by conventional oral administration in order to obtain relevant effects on coronary flow. A comparable effect on the brachial artery flow-mediated vasodilation was shown by Lekakis with an oral dose of $5 \mathrm{mg}$ of nebivolol [23].

Differences in response to nebivolol between coronary artery disease and control group

In controls, endothelial function is normal with a normal coronary flow reserve value. In coronary artery disease patients, endothelial dysfunction is present and coronary flow reserve is reduced. Stimulation of the endothelium by nebivolol acts mainly in the diseased arteries with an increase in maximal coronary flow and almost normalization of coronary flow reserve. In the normal coronary artery, the beta-blocking effect of nebivolol predominates rather than the vasodilating effect on maximal flow. Thus, in the presence of normal endothelial function, the beta-blocking effect of nebivolol becomes evident, whereas in the diseased vessel the vasodilating effect of nebivolol prevails. These changes are reflected by the changes in coronary peripheral resistance with an increase in resistance in the controls at rest and a decreased resistance in coronary artery disease patients under hyperemic conditions.

The anti-ischemic effect of nebivolol in the presence of coronary artery disease is based on the following mechanisms: (1) reduction in oxygen demand through the $\beta 1$-adrenoreceptor blocking properties as reflected by the decrease in heart rate and rate-pressure product, (2) maintained (not diminished) coronary flow at rest, (3) increased coronary flow reserve through the increase in hyperemic flow (=increased oxygen supply).
These three mechanisms influence the oxygen supplydemand balance of the myocardium favorably.

Collateral flow index and nebivolol The coronary collateral flow decreased significantly after nebivolol. Previous reports have shown a similar response of coronary collateral flow to the selective $\beta 1$-blocker metoprolol [24]. The reduction in coronary collateral flow has been attributed to a vasoconstrictive response of the collateral vessel to metoprolol with an increase in vascular resistance. With nebivolol a similar response was observed with a decrease in coronary collateral perfusion. However, there was a close relation between heart rate and changes in collateral flow. This finding supports the hypothesis that collateral flow is mainly determined by the requirements of the collateral-dependent myocardium.

Limitations Several limitations have to be considered for this study:

(1) Changes in heart rate and mean aortic pressure may affect coronary flow and flow velocity. In controls, baseline flow decreased after nebivolol administration, and the rise in coronary flow reserve was attributed to the reduction in coronary flow at rest in the presence of a maintained hyperemic flow. In coronary artery disease patients, however, the decrease in heart rate and mean aortic pressure after nebivolol was not associated with a change in resting coronary flow. This finding could be explained by the beneficial effect of nebivolol on nitric oxide-release which may counteract the flow reduction induced by the presence of endothelial dysfunction in coronary artery disease patients.

(2) Comparisons with other selective $\beta 1$-adrenoreceptor antagonists (i.e., metoprolol) were not performed. Previous studies from our group demonstrated a similar response of coronary flow and reserve capacity [12] as well as collateral flow [24] to metoprolol. However, study protocols differ substantially, and therefore, data may not be extrapolated for comparison purposes.

(3) The control group consisted of patients who underwent coronary angiography to exclude the presence of coronary artery disease. Therefore, the results of the control group do not reflect the response to nebivolol in normals.

(4) There was an imbalance between the two groups with regard to smoking and medication with ACE inhibitors. We therefore performed an multivariable regression analysis with covariates to determine the effect of medication and presence of risk factors (smoking, diabetes) on the effect of nebivolol on 
coronary flow and flow reserve. The analysis of variance showed no effect of cardiovascular risk factors as well as baseline medication including ACE-inhibitors on the results obtained.

(5) Coronary flow reserve may have been affected by other factors apart form endothelial function such as left ventricular mass and cholesterol values. Cholesterol values were similar in the two study groups. Left ventricular mass was not determined and thus no comment on this relationship can be made, except that the incidence of hypertension was similar in both groups.

(6) The concomitant use of beta-blockers in the two groups may have affected the results of the present study. The only beta-blocker used was metoprolol and in a similar percentage in both groups. However, all cardiovascular medication was stopped at least 48 $\mathrm{h}$ prior to the study and all patients showed decrease in heart rate under nebivolol.

\section{Conclusions}

Nebivolol is associated with a significant increase in coronary flow reserve in controls as well as in patients with coronary artery disease. In controls, the rise in coronary flow reserve is mainly attributed to the decrease in resting coronary flow ( $\beta$-blocking effect), whereas in coronary artery disease patients, this effect is mainly due to an increase in maximal coronary flow during adenosine infusion without a change in resting flow. The increase in coronary flow (supply $\uparrow$ ) and the reduction in rate-pressure product (demand $\downarrow$ ) after nebivolol explain the excellent anti-ischemic properties of this compound in patients with coronary artery disease. Coronary flow index decreased with nebivolol parallel to the reduction in oxygen requirements of the collateral-dependent myocardium.

Acknowledgements Dr Francesco Vigorito was supported by an educational grant of Menarini S.A., Firenze, Italy.

\section{References}

1. Hilton R, Eicholtz F. The influence of chemical factors on the coronary circulation. J Physiol 1925;59:413-25.

2. Katz LN, Lindner E. Quantitative relation between reactive hyperemia and the myocardial ischemia which it follows. Am J Physiol 1939;126:283-8.

3. Coffman JD, Gregg DE. Reactive hyperemia characteristics of the myocardium. Am J Physiol 1960;199:1143-9.
4. Marcus M, Wright C, Doty D, Eastham C, Laughlin D, Krumm P, et al. Measurements of coronary velocity and reactive hyperemia in the coronary circulation of humans. Circ Res 1981;49(4):877-91.

5. Bookstein JJ, Higgins CB. Comparative efficacy of coronary vasodilatory methods. Invest Radiol 1977;12(2):121-7.

6. Christensen CW, Rosen LB, Gal RA, Haseeb M, Lassar TA, Port SC. Coronary vasodilator reserve. Comparison of the effects of papaverine and adenosine on coronary flow, ventricular function, and myocardial metabolism. Circulation 1991;83(1):294-303.

7. Wilson RF, Wyche K, Christensen BV, Zimmer S, Laxson DD. Effects of adenosine on human coronary arterial circulation. Circulation 1990;82(5):1595-606.

8. Rossen JD, Quillen JE, Lopez AG, Stenberg RG, Talman CL, Winniford MD. Comparison of coronary vasodilation with intravenous dipyridamole and adenosine. J Am Coll Cardiol 1991;18(2):485-91.

9. Felder L, Vassalli G, Vassalli F, Jiang Z, Grimm J, Krayenbuehl HP, et al. Clinical significance of coronary flow reserve: effect of papaverine and exercise. Coron Artery Dis 1994;5(4):347-58.

10. Marcus ML, Chilian WM, Kanatsuka H, Dellsperger KC, Eastham CL, Lamping KG. Understanding the coronary circulation through studies at the microvascular level. Circulation 1990;82(1):1-7.

11. Bottcher M, Czernin J, Sun K, Phelps ME, Schelbert HR. Effect of beta 1 adrenergic receptor blockade on myocardial blood flow and vasodilatory capacity. J Nucl Med 1997;38(3):442-6.

12. Billinger M, Seiler C, Fleisch M, Eberli FR, Meier B, Hess OM. Do beta-adrenergic blocking agents increase coronary flow reserve? J Am Coll Cardiol 2001;38(7):1866-71.

13. Dessy C, Moniotte S, Ghisdal P, Havaux X, Noirhomme P, Balligand JL. Endothelial beta3-adrenoceptors mediate vasorelaxation of human coronary microarteries through nitric oxide and endothelium-dependent hyperpolarization. Circulation 2004;110(8):948-54.

14. Doucette JW, Corl PD, Payne HM, Flynn AE, Goto M, Nassi M, et al. Validation of a Doppler guide wire for intravascular measurement of coronary artery flow velocity. Circulation 1992;85(5):1899-911.

15. Dawes M, Brett SE, Chowienczyk PJ, Mant TG, Ritter JM. The vasodilator action of nebivolol in forearm vasculature of subjects with essential hypertension. Br J Clin Pharmacol 1999;48(3):460-3.

16. Young MA, Vatner DE, Vatner SF. Alpha- and betaadrenergic control of large coronary arteries in conscious calves. Basic Res Cardiol 1990;85(Suppl 1):97-109.

17. Robertson RM, Wood AJ, Vaughn WK, Robertson D. Exacerbation of vasotonic angina pectoris by propranolol. Circulation 1982;65(2):281-5.

18. Kern MJ, Ganz P, Horowitz JD, Gaspar J, Barry WH, Lorell $\mathrm{BH}$, et al. Potentiation of coronary vasoconstriction by betaadrenergic blockade in patients with coronary artery disease. Circulation 1983;67(6):1178-85.

19. Dessy C, Saliez J, Ghisdal P, Daneau G, Lobysheva II, Frerart F, et al. Endothelial beta3-adrenoreceptors mediate nitric oxide-dependent vasorelaxation of coronary microvessels in response to the third-generation beta-blocker nebivolol. Circulation 2005;112(8):1198-205.

20. Flather MD, Shibata MC, Coats AJ, Van Veldhuisen DJ, Parkhomenko A, Borbola $\mathrm{J}$, et al. Randomized trial to determine the effect of nebivolol on mortality and cardiovascular hospital admission in elderly patients with heart failure (SENIORS). Eur Heart J 2005;26(3):215-25. 
21. Stoleru L, Wijns W, van Eyll C, Bouvy T, Van Nueten L, Pouleur H. Effects of D-nebivolol and L-nebivolol on left ventricular systolic and diastolic function: comparison with D-L-nebivolol and atenolol. J Cardiovasc Pharmacol 1993;22(2):183-90.

22. Galderisi M, Cicala S, D'Errico A, de Divitiis O, de Simone G. Nebivolol improves coronary flow reserve in hypertensive patients without coronary heart disease. J Hypertens 2004;22(11):2201-8.
23. Lekakis JP, Protogerou A, Papamichael C, Vamvakou G, Iconomidis I, Fici F, et al. Effect of nebivolol and atenolol on brachial artery flow-mediated vasodilation in patients with coronary artery disease. Cardiovasc Drugs Ther 2005; 19(4):277-81.

24. Billinger M, Raeber L, Seiler C, Windecker S, Meier B, Hess $\mathrm{OM}$. Coronary collateral perfusion in patients with coronary artery disease: effect of metoprolol. Eur Heart J 2004;25(7):565-70. 